\title{
The effect of an antiserotonin agent pizotifen on platelet aggregability in migraine patients
}

\author{
S MAZAL AND E A RACHMILEWITZ
}

From the Out-Patient Neurological Clinic, Hadassah University Hospital, Headache Clinic, Misgav Ladach General Hospiral, and Haematology Service, Hadassah University Hospital, Mount Scopus, Hebrew University, Hadassah University Medical School, Jerusalem, Israel

SUMMARY In 19 patients who were suffering from frequent migraine attacks and were treated with pizotifen, spontaneous platelet aggregation (SPA) was measured prior to treatment and at the second, fourth and sixth month of treatment. A Migraine Headache Score (MHS) was employed for evaluation of the "activity" of migraine in the same periods. Spontaneous platelet aggregation was also measured in a control group of $\mathbf{4 0}$ migraine-free subjects. Prior to treatment the mean value of SPA in the group of migraine patients was four times higher than in the control group. In the 19 migraine patients the mean value of SPA in the second month of treatment showed a $48 \%$ reduction when compared with the mean value of SPA prior to treatment. Clear clinical improvement was observed during the treatment, and the average MHS in the second month of treatment showed a $77 \%$ reduction compared with the average MHS prior to treatment. The increased rate of SPA prior to treatment and the close correlation between the SPA rate and "activity" of migraine during the treatment suggest that increased platelet aggregability may play a significant role in the pathogenesis of migraine.

Curran, Hinterberger and Lance ${ }^{1}$ found that total plasma serotonin falls sharply at the onset of migraine attacks and remains low for the duration of the attack, gradually returning thereafter to pre-headache level. Since blood serotonin is carried almost entirely by blood platelets, ${ }^{2}$ and since serotonin is one of the substances capable of inducing platelet aggregation, attention was drawn to platelet aggregability changes in migraine patients. Hilton and Cumings $^{3}{ }^{4}$ found that the aggregation response of the platelets to serotonin was increased in migraine patients in the headache-free intervals when compared to the aggregation response to serotonin in normal subjects. They also found that methysergide, a chemical agent with potent antiserotonin properties, which was found effective in preventive treatment of migraine, modified the aggregation response to serotonin in migraine patients causing significant reduction of serotonin-induced platelet aggregation.

Pizotifen, a tricyclic chemical compound with

Address for reprint requests: Dr S Mazal, 7 Etzel Street, French Hill, Jerusalem, Israel.

Accepted 16 July 1980 powerful antiserotonin and antihistamine properties, significantly reduced the frequency and severity of migraine attacks. ${ }^{5-7}$ The aim of this study was to evaluate the effect of pizotifen on platelet aggregability in migraine patients.

\section{Patients and methods}

Patients with at least four attacks of migraine per month during the three months prior to treatment were selected for preventive treatment with pizotifen. Criteria for diagnosing migraine were those described by the Ad Hoc Committee on Classification of Headache. ${ }^{8}$

Each patient had a detailed general medical and neurological evaluation. General physical examination, routine blood tests and urine analysis were done in every case to exclude diabetes, hyperlipidaemia, liver and kidney diseases, and obvious haematological abnormalities. Detailed personal and family history was obtained in every case and patients who suffered in the past from thrombophlebitis or any other occlusive vascular events were excluded from the study. Patients who received any medication that contained oestrogens or progesterone within one year prior to the beginning of the study were also excluded. None of the patients who entered the study received any regular medication within three months 
prior to the beginning of the study. An EEG, echoencephalogram and isotope brain scan were done in each patient. The results of the isotope scans and echoencephalograms were normal in all the 19 patients who entered the study. The EEGs were normal in 15 patients; in three cases occasional irregular sharp waves were recorded from the posterior regions of the brain and in one case on repeated EEG examinations a sharp focus appeared in the right parietal area and in the left parietal area alternatively. In four patients whose headaches were accompanied by transient focal neurological symptoms computed tomography of the head also was performed, and the results were normal in all cases. The age of the 19 patients ranged from 10 to 60 years. There were eight men and 11 women. Four had classic migraine and 15 had common migraine according to the classification of the Ad Hoc Committee on Classification of Headache. $^{8}$ The patients received pizotifen (Sandomigran, Sandoz) $1.5 \mathrm{mg}$ daily (divided into 3 doses of $0.5 \mathrm{mg}$ each), except one patient-a 10 year old boy, who received $1 \mathrm{mg}$ daily $(0.5 \mathrm{mg}$ twice a day).

The spontaneous platelet aggregation (SPA) test was done in every patient within four weeks prior to the beginning of the treatment by pizotifen, and was repeated at the second, fourth and sixth month of the treatment. The method described by Holdrinet et $a^{9}$ was used to determine the SPA. The method is based on the fact that platelet-rich plasma prepared by centrifugation from heparinised blood (heparin-PRP) invariably contains a smaller number of platelets than platelet-rich plasma prepared in the same way from EDTA-blood (EDTA-PRP). ${ }^{10}$ It is supposed $^{10}$ that this difference is caused by sedimentation during centrifugation of platelet aggregates which are spontaneously formed in heparinised blood and are absent in EDTA-blood. Therefore, the degree of spontaneous aggregation can be measured by comparing the platelet number in EDTA-PRP with the platelet number in heparinPRP. 9

Blood was obtained by venipuncture using disposable needles. Four $\mathrm{ml}$ of blood was collected in each of two plastic tubes. One contained $0.4 \mathrm{ml}$ disodium-EDTA solution $(4 \%)$, and the second contained $0.4 \mathrm{ml}$ veronal buffer with $20 \mathrm{u}$ heparin. The tubes were immediately centrifuged at room temperature at $150 \mathrm{~g}$ for $10 \mathrm{~min}$. The platelet concentration in the supernatant plasma of each of the two samples was then determined by visual counting under a microscope. The difference between the platelet concentration in EDTA-PRP and the platelet concentration in heparin-PRP, expressed as per cent of the platelet concentration in EDTA-PRP, was accepted as the measurement of spontaneous aggregation. The test was always completed within one hour from the time of the venipuncture. The normal level of SPA in our laboratory was established by testing 40 control subjects (21 women and 19 men) aged from 18 to 59 years. The control subjects were drawn from the office staff of a medical insurance company. They were interviewed before the SPA test, and those included in the control group did not suffer from diabetes, liver diseases or haematological diseases. They did not have a history of occlusive vascular events, and never suffered from migraine attacks. They did not use any medication within seven days prior to the test. The women included in the control group were not pregnant at the time of the test, and did not use oral contraceptives within 12 months before the test. In this group the results ranged from $4 \%$ to $23.8 \%$ with a mean value of $14 \cdot 7 \pm 5 \cdot 1 \%$, and with normal distribution.

Each month during three months prior to the beginning of the treatment, and during the whole period of the treatment with pizotifen, all the patients were required to complete a special form where the dates of the attacks and their duration in hours were recorded. For estimation of the "activity" of migraine, a Migraine Headache Score (MHS) was calculated for every month by adding together the number of hours of all the attacks of migraine headache suffered by the patient during this month.

\section{Results}

The method of measuring SPA was reproducible. When SPA was determined in four patients for three consecutive days prior to treatment the following results were obtained: $79.4 \%, 85 \%, 82 \%$ in subject $A ; 75 \%, 68.5 \%, 71 \%$ in subject $B$; $60 \%, 67 \%, 58 \%$ in subject $C ; 72 \% 65 \%$, $68 \%$ in subject $\mathrm{D}$.

Prior to treatment the SPA was above 25\% (control group's mean value $+2 \mathrm{SD}$ ) in 17 of the 19 patients (see figure). The mean value of SPA in the group of patients prior to treatment was four times higher than in the control group $(p<0.001)$. The mean value of SPA of the 19 patients in the second month of treatment showed a $48 \%$ reduction when compared with the mean value of SPA for the same group in the month prior to treatment (table 1) $(\mathrm{p}<0.001)$. The mean values of SPA in the fourth and sixth month of treatment showed a further reduction; however, the difference between the mean values of SPA of the second and fourth, and fourth and sixth months was not statistically significant.

Table 1 Spontaneous platelet aggregation in migraine patients before and during treatment with pizotifen

\begin{tabular}{|c|c|c|c|}
\hline $\begin{array}{l}\text { Before } \\
\text { treatment } \\
\%\end{array}$ & $\begin{array}{l}\text { 2nd month } \\
\text { of treatment } \\
\%\end{array}$ & $\begin{array}{l}4 \text { th month } \\
\text { of treatment } \\
\%\end{array}$ & $\begin{array}{l}6 \text { th month } \\
\text { of treatment } \\
\%\end{array}$ \\
\hline $\begin{array}{l}60 \cdot 9 \pm 22 \cdot 8^{*} \\
(19)\end{array}$ & $\begin{array}{l}30 \cdot 4 \pm 19 \cdot 4^{*} \\
(19)\end{array}$ & $\begin{array}{l}23 \cdot 2 \pm 20 \cdot 3 * \\
(17)\end{array}$ & $\begin{array}{l}14 \cdot 2 \pm 5 \cdot 9^{*} \\
\text { (11) }\end{array}$ \\
\hline
\end{tabular}

Number of patients in parentheses. ${ }^{*}$ Mean result $\pm \mathrm{SD}$. 


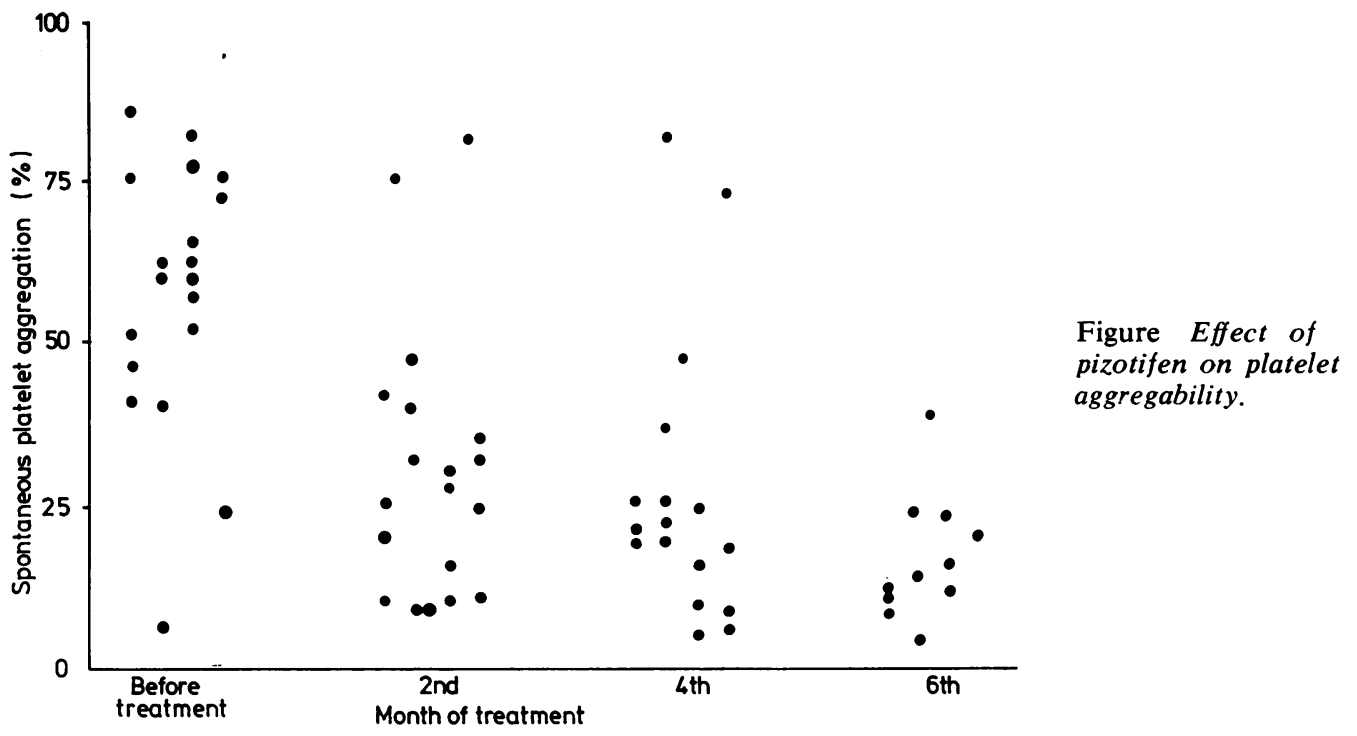

Clear clinical improvement was seen during the treatment. This was expressed by a $77 \%$ reduction in the average number of hours of migraine headache observed already in the second month of treatment compared to the month prior to treatment (table 2) $(\mathrm{p}<0.001)$. When the average MHS of the second and fourth, and fourth and sixth months of treatment were compared, the difference was not statistically significant.

Table 2 Average migraine headache score before and during treatment with pizotifen

\begin{tabular}{llll}
\hline $\begin{array}{l}\text { Before } \\
\text { treatment }\end{array}$ & $\begin{array}{l}\text { 2nd month } \\
\text { of treatment }\end{array}$ & $\begin{array}{l}\text { 4th month } \\
\text { of treatment }\end{array}$ & $\begin{array}{l}\text { 6th month } \\
\text { of treatment }\end{array}$ \\
\hline $84 \cdot 3 \pm 26 \cdot 7^{*}$ & $20 \cdot 1 \pm 24 \cdot 5^{*}$ & $22 \cdot 5 \pm 28 \cdot 0^{*}$ & $15 \cdot 9 \pm 8 \cdot 3^{*}$ \\
$(19)$ & $(19)$ & $(17)$ & $(11)$
\end{tabular}

Number of patients in parentheses. ${ }^{*}$ Mean result \pm SD.

As seen from tables 1 and 2, 11 of the 19 patients continued to receive the treatment during the whole six months period. Eight patients did not complete the six months period. The reason for the early discontinuation of the treatment in most of the cases was complete freedom from headaches or a significant reduction in their frequency usually observed already at the second month of treatment. Only in two patients was the medication stopped because of lack of improvement.

\section{Discussion}

Kalendovsky and Austin ${ }^{11}$ studied platelet aggregability in patients with different types of migraine and in normal subjects, and found that patients with migraine as a group clearly had more aggregable platelets than did control subjects. They suggested that increased platelet aggregability correlates with certain neurological symptoms which accompany migraine attacks, since in their study platelet aggregability was found to be increased more frequently and to a greater extent in the group of patients with complicated migraine, when compared to the group of patients with uncomplicated migraine. Recognising that vasoconstriction is an important factor that contributes to the disturbances of the cerebral perfusion in migraine patients, these authors suggested an additional mechanism which can further compromise to a significant degree the cerebral perfusion in patients with complicated migraine. They postulated that small vascular occlusions by small "white" platelet thrombi may appear in patients with complicated migraine as a result of the hyperaggregability of their platelets. These authors also suggested that the use of oestrogens causes a further increase in platelet aggregability in migraine sufferers. ${ }^{12}$

We reported a case where a clear correlation was seen between the frequency of migraine attacks induced by oral contraceptives and the rate of platelet aggregability, and where a decrease in the frequency of migraine attacks and their eventual disappearance was observed after treatment with platelet inhibitors. ${ }^{13} \mathrm{We}$ suggested in that article that increased platelet 
aggregability may play a significant role in the pathogenesis of the "migraine syndrome" that appears during the course of oral contraception, as well as in the pathogenesis of the idiopathic or familial migraine disease. Contrary to these observations, Couch and Hassanein, ${ }^{14}$ who also found that the aggregability of platelets is significantly increased in migraine patients, did not find any correlation between the level of the platelet hyperaggregability and between the level of "activity" of migraine or the neurological symptoms accompanying the migraine attacks. Nor did they find any significant difference in platelet aggregability between those female migraine patients who used birth control pills and between those who did not use them. According to these authors the increased platelet aggregability is a concomitant feature in migraine subjects, unrelated to the actual migraine process, although it can help explain the increased incidence of stroke and heart attack in migraine patients.

The results of the present study show that there does exist a clear correlation between the "activity" of migraine, expressed by the MHS, and the rate of SPA. However, it is still not clear if the observed changes in platelet aggregability are one of the causes or one of the results of the clinical improvement that appears during treatment with pizotifen. Further investigations need to be undertaken in order to find out whether increased platelet aggregability is one of the basic mechanisms involved in the pathogenesis of migraine or if it is only a concomitant "epiphenomenon." However, the close correlation between the "activity" of migraine and the rate of platelet aggregation shown in this study and the encouraging results of the use of platelet antagonists for prophylaxis of migraine attacks ${ }^{13} 15$ seem to support the first possibility rather than the second. In two of the 19 patients the SPA rate was normal prior to treatment and remained almost unchanged during the course of the treatment. Kalendovsky and Austin ${ }^{11}$ also found normal platelet aggregability in approximately $35 \%$ of their migraine patients, while in $65 \%$ the platelet aggregability was increased. It is possible that the groups of migraine patients studied by us and by Kalendovsky and Austin may not be homogeneous concerning the pathogenesis of their disease.

We thank Mrs Sarah Sagih for the perform- ance of the platelet aggregation tests, and Mrs Shila Ben-Tuvia and Mrs Tzofnat Koelewyn for statistical analysis.

\section{References}

1 Curran DA, Hinterberger H, Lance JW. Total plasma serotonin, 5-hydroxyindolacetic acid and p-hydroxy-m-methoxymandelic acid excretion in normal and migrainous subjects. Brain 1965; 88:997-1010.

2 Hardisty RM, Stacey RS. 5-hydroxytryptamine in normal human platelets. J Physiol 1955; 130: 711-20.

3 Hilton BP, Cumings JN. An assessment of platelet aggregation induced by 5-hydroxytryptamine. J Clin Pathol 1971; 24:250-8.

4 Hilton BP, Cumings JN. 5-hydroxytryptamine levels and platelet aggregation responses in subjects with acute migraine headache. $J$ Neurol Neurosurg Psychiatry 1972; 35:505-9.

5 Carroll JD, Maclay WP. Pizotifen (BC-105) in migraine prophylaxis. Curr Med Res Opin 1975; 3:68-71.

6 Lawrence ER, Hossain M, Littlestone W. Sandomigran for migraine prophylaxis; controlled multicenter trial in general practice. Headache 1977; 17:109-12.

7 Mikropoulos HE. Toleration and effectiveness of pizotifen in migraine. Res Clin Stud Headache 1978; 6:167-72.

8 Ad Hoc Committee on Classification of Headache. Classification of headache. JAMA 1962; 179:717-8.

9 Holdrinet A, Ewals M, Haanen C. A simple test for measuring platelet aggregation. Thromb Haemost 1969; 22:174-91.

10 Mannucci PM, Sharp AA. Platelet volume and shape in relation to aggregation and adhesion. Br J Haematol 1967; 13:604-17.

11 Kalendovsky Z, Austin JH. Complicated migraine: Its association with increased platelet aggregability and abnormal plasma coagulation factors. Headache 1975; 15:18-35.

12 Kalendovsky Z, Austin JH. Changes in blood clotting systems during migraine attacks. Headache 1977; 16:293-312.

13 Mazal S. Migraine attacks and increased platelet aggregability induced by oral contraceptives. A ust NZ J Med 1978; 8:646-8.

14 Couch JR, Hassanein RS. Platelet aggregability in migraine. Neurology (Minneap) 1977; 27: 843-8.

15 Dalessio DJ, Otis S, Smith R. Vasomotor phenomena, platelet antagonism and migraine therapy. Res Clin Stud Headache 1978; 6:34-40. 